\title{
Automatic Differentiation for Nonlinear Controller Design
}

\author{
Klaus Röbenack \\ Institut für Regelungs- und Steuerungstheorie \\ TU Dresden, Mommsenstr. 13, D-01062 Dresden, Germany \\ klaus@roebenack.de \\ http://www.roebenack.de
}

\begin{abstract}
Several new algorithms for nonlinear controller design are based on differential-geometric concepts. Up to now, the feedback of the controller has been computed symbolically. The author proposes a method to compute the feedback using automatic differentiation. With this approach, time-consuming symbolic computation can be avoided.
\end{abstract}

\section{Introduction}

During the last decades, several methods for nonlinear controller design based on differential geometry have been developed [1-3]. One import approach is the exact linearization via feedback [2]. This method has been successfully applied to a large number of real-world systems [4-6]. Up to now, the derivatives required by these algorithms have been computed symbolically using computer algebra packages [7-9]. The use of these methods is limited due to a burden of symbolic computations involved [10]. These disadvantages can be circumvented with automatic differentiation. Moreover, the methods presented here can be applied to algorithmic descriptions of the plant to be controlled. This is especially useful since complicated systems are constructed using either traditional programming languages, e.g., $\mathrm{C}++$ in fast circuit simulation [11], or modeling languages such as Modelica $[12,13]$ or VHDL-AMS $[14,15]$.

In Sect. 2 some facts of computing Taylor coefficients by automatic differentiation are given. In Sect. 3, we review controller design for nonlinear state-space systems. The use of automatic differentiation is described in Sect. 4. The design of so-called tracking controllers is addressed in Sect. 5 .

\section{Univariate Taylor Series}

Consider a smooth map $\mathbf{F}: \mathbb{R}^{n} \rightarrow \mathbb{R}^{m}$ which maps a curve

$$
\mathbf{x}(t)=\mathbf{x}_{0}+\mathbf{x}_{1} t+\cdots+\mathbf{x}_{d} t^{d}+O\left(t^{d+1}\right)
$$

of the vector space $\mathbb{R}^{n}$ into a curve

$$
\mathbf{z}(t)=\mathbf{F}(\mathbf{x}(t))=\mathbf{z}_{0}+\mathbf{z}_{1} t+\cdots+\mathbf{z}_{d} t^{d}+O\left(t^{d+1}\right)
$$

P.M.A. Sloot et al. (Eds.): ICCS 2002, LNCS 2330, pp. 1059-1068, 2002.

(C) Springer-Verlag Berlin Heidelberg 2002 
of $\mathbb{R}^{m}$ with

$$
\mathbf{z}_{j}=\left.\frac{1}{j !} \frac{\partial^{j} \mathbf{z}(t)}{\partial t^{j}}\right|_{t=0}
$$

Each Taylor coefficient $\mathbf{z}_{j} \in \mathbb{R}^{m}$ is uniquely determined by the coefficients $\mathbf{x}_{0}, \ldots, \mathbf{x}_{j} \in \mathbb{R}^{n}$. In particular, we have

$$
\begin{aligned}
& \mathbf{z}_{0}=\mathbf{F}\left(\mathbf{x}_{0}\right) \\
& \mathbf{z}_{1}=\mathbf{F}^{\prime}\left(\mathbf{x}_{0}\right) \mathbf{x}_{1} \\
& \mathbf{z}_{2}=\mathbf{F}^{\prime}\left(\mathbf{x}_{0}\right) \mathbf{x}_{2}+\frac{1}{2} \mathbf{F}^{\prime \prime}\left(\mathbf{x}_{0}\right) \mathbf{x}_{1} \mathbf{x}_{1} \\
& \mathbf{z}_{3}=\mathbf{F}^{\prime}\left(\mathbf{x}_{0}\right) \mathbf{x}_{3}+\mathbf{F}^{\prime \prime}\left(\mathbf{x}_{0}\right) \mathbf{x}_{1} \mathbf{x}_{2}+\frac{1}{6} \mathbf{F}^{\prime \prime \prime}\left(\mathbf{x}_{0}\right) \mathbf{x}_{1} \mathbf{x}_{1} \mathbf{x}_{1}
\end{aligned}
$$

Using automatic differentiation, the Taylor coefficients $\mathbf{z}_{0}, \ldots, \mathbf{z}_{d}$ can be obtained without symbolic computations of the high-dimensional derivative tensors $\mathbf{F}^{\prime} \in \mathbb{R}^{m \times n^{2}}, \mathbf{F}^{\prime \prime} \in \mathbb{R}^{m \times n^{3}}, \ldots, \mathbf{F}^{(d)} \in \mathbb{R}^{m \times n^{d}}$. The tools ADOL-C [16] and TADIFF [17] use the forward mode to compute this Taylor coefficients. For many functions such as exp, sin, cos, arctan, tanh, the number of operations to be performed is bounded by the square of the degree, $d$, of the Taylor coefficients, i.e., $\operatorname{OPS}\left\{\mathbf{z}_{0}, \ldots, \mathbf{z}_{d}\right\} \lesssim d^{2} \cdot \operatorname{OPS}\left\{\mathbf{F}\left(\mathbf{x}_{0}\right)\right\}$, see [18, Sect. 10.2].

\section{Exact Linearization via Feedback}

In this section we consider the design of a controller for nonlinear input-affine state-space systems

$$
\dot{\mathbf{x}}=\mathbf{f}(\mathbf{x})+\mathbf{g}(\mathbf{x}) u, \quad y=h(\mathbf{x}) .
$$

The maps $\mathbf{f}, \mathbf{g}: \mathbb{R}^{n} \rightarrow \mathbb{R}^{n}$ and $h: \mathbb{R}^{n} \rightarrow \mathbb{R}$ are assumed to be sufficiently smooth. We need Lie derivatives $L_{\mathbf{f}} h(\mathbf{x})=\frac{\partial h}{\partial \mathbf{x}} \mathbf{f}(\mathbf{x})$ of $h$ along $\mathbf{f}$, see [2]. Higher order Lie derivatives $L_{\mathbf{f}}^{k} h(\mathbf{x})$ are defined by

$$
L_{\mathbf{f}}^{k} h(\mathbf{x})=\frac{\partial L_{\mathbf{f}}^{k-1} h(\mathbf{x})}{\partial \mathbf{x}} \mathbf{f}(\mathbf{x}) \quad \text { with } \quad L_{\mathbf{f}}^{0} h(\mathbf{x})=h(\mathbf{x}) .
$$

Similarly, the mixed Lie derivative $L_{\mathbf{g}} L_{\mathbf{f}}^{k} h(\mathbf{x})$ is given by

$$
L_{\mathbf{g}} L_{\mathbf{f}}^{k} h(\mathbf{x})=\frac{\partial L_{\mathbf{f}}^{k} h(\mathbf{x})}{\partial \mathbf{x}} \mathbf{g}(\mathbf{x})
$$

First, let us consider the case of an autonomous system

$$
\dot{\mathbf{x}}=\mathbf{f}(\mathbf{x}), \quad y=h(\mathbf{x})
$$

The time derivatives of the output can be written as

$$
\dot{\mathbf{y}}=\frac{\partial h}{\partial \mathbf{x}} \mathbf{f}(\mathbf{x})=L_{\mathbf{f}} h(\mathbf{x}), \ddot{\mathbf{y}}=\frac{\partial L_{\mathbf{f}} h}{\partial \mathbf{x}} \mathbf{f}(\mathbf{x})=L_{\mathbf{f}}^{2} h(\mathbf{x}), \ldots, y^{(k)}=L_{\mathbf{f}}^{k} h(\mathbf{x})
$$


For an initial value $\mathbf{x}_{0}=\mathbf{x}(0)$, the output signal $y$ is expressed by the series expansion

$$
y(t)=\sum_{i=0}^{\infty} L_{\mathbf{f}}^{i} h\left(\mathbf{x}_{0}\right) \frac{t^{i}}{i !}
$$

see [2, p. 140] for details.

Now, we will take the input $u$ into consideration. According to [2], the system (3) is said to have relative degree $r$ at $\mathbf{x}_{0} \in \mathbb{R}^{n}$ if $L_{\mathbf{g}} L_{\mathbf{f}}^{k} h(\mathbf{x})=0$ for all $\mathbf{x}$ in a neighborhood of $\mathbf{x}_{0}$ and all $k \in\{0, \ldots, r-2\}$, and $L_{\mathbf{g}} L_{\mathbf{f}}^{r-1} h\left(\mathbf{x}_{0}\right) \neq 0$. Then, the time derivatives of the output of (3) are

$$
y=L_{\mathbf{f}}^{0} h(\mathbf{x}), \ldots, y^{(r-1)}=L_{\mathbf{f}}^{r-1} h(\mathbf{x}), y^{(r)}=L_{\mathbf{f}}^{r} h(\mathbf{x})+L_{\mathbf{g}} L_{\mathbf{f}}^{r-1} h(\mathbf{x}) .
$$

A case of particular interest is $r=n$. If the map $\Phi$ defined by $\xi_{i}=\phi_{i}(\mathbf{x})=$ $L_{\mathbf{f}}^{i-1} h(\mathbf{x})$ for $i=1, \ldots, n$ is a diffeomorphism, the change of coordinates $\boldsymbol{\xi}=\Phi(\mathbf{x})$ with $\boldsymbol{\xi}=\left(\xi_{1}, \ldots, \xi_{n}\right)^{T}$ transforms (3) into the prime form [19]

$$
\dot{\xi}_{1}=\xi_{2}, \ldots, \dot{\xi}_{n-1}=\xi_{n}, \dot{\xi}_{n}=L_{\mathbf{f}}^{n} h(\mathbf{x})+L_{\mathbf{g}} L_{\mathbf{f}}^{n-1} h(\mathbf{x}) u, \quad y=\xi_{1} .
$$

If we use the state feedback

$$
u=\frac{1}{L_{\mathbf{g}} L_{\mathbf{f}}^{n-1} h(\mathbf{x})}\left[-L_{\mathbf{f}}^{n} h(\mathbf{x})-\mathbf{k}^{\top} \boldsymbol{\xi}+v\right]
$$

with $\mathbf{k}=\left(k_{0}, \ldots, k_{n-1}\right)^{T}$ and the new input $v$, one obtains a linear time-invariant system

$$
\dot{\xi}_{1}=\xi_{2}, \ldots, \dot{\xi}_{n-1}=\xi_{n}, \dot{\xi}_{n}=-\sum_{i=1}^{n} k_{i-1} \xi_{i}+v, \quad y=\xi_{1}
$$

in the new coordinates. The dynamical behavior of (10) is prescribed by its characteristic polynomial $\rho(\lambda)=k_{0}+k_{1} \lambda+k_{2} \lambda^{2}+\cdots+k_{n-1} \lambda^{n-1}+\lambda^{n}$. We can apply methods of linear control theory [20] for the choice of the coefficients $k_{i}$. Fig. 1 shows the control scheme.

In general, the relative degree $r$ may be less than $n$. In terms of the original coordinates, the feedback (9) is given by

$$
u=-\frac{1}{L_{\mathbf{g}} L_{\mathbf{f}}^{r-1} h(\mathbf{x})}\left[k_{0} L_{\mathbf{f}}^{0} h(\mathbf{x})+\cdots+k_{r-1} L_{\mathbf{f}}^{r-1} h(\mathbf{x})+L_{\mathbf{f}}^{r} h(\mathbf{x})-v\right] .
$$

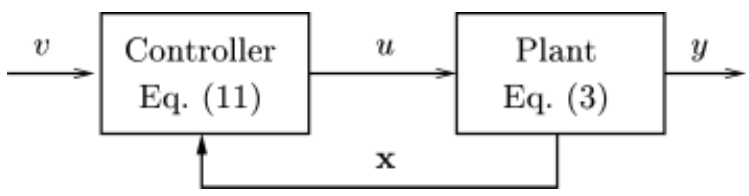

Fig. 1. Control scheme for exact linearization via feedback 


\section{Computation of Lie Derivatives}

A symbolic computation of the Lie derivatives needed in (11) may result in extremely large expressions [10]. In fact, the size of the expressions may increase exponentially in the order of the Lie derivatives. Using automatic differentiation, the computational effort to compute the function values of Lie derivatives increases at most quadratically.

Let $r$ denote the relative degree of (3). First, we will compute a series expansion of the autonomous system (6). We consider the expansion (1) of the solution of the ODE and a map $\mathbf{z}=\mathbf{f}(\mathbf{x})$ with the series expansion of $\mathbf{z}$ such as in (2). Since $\mathbf{x}$ is assumed to be the solution of (6), we have $\mathbf{z} \equiv \dot{\mathbf{x}}$ and $\mathbf{x}_{i+1}=\mathbf{z}_{i} /(i+1)$ for $i \geq 0$. Starting with an initial value $\mathbf{x}_{0} \in \mathbb{R}^{n}$, we compute the function value $\mathbf{z}_{0}$ and obtain $\mathbf{x}_{1}=\mathbf{z}_{0}$. Using $\mathbf{x}_{0}$ and $\mathbf{x}_{1}$, the Taylor coefficient $\mathbf{z}_{1}$ can be obtained by a forward sweep of automatic differentiation. We repeat this procedure until we get the Taylor coefficients $\mathbf{x}_{0}, \ldots, \mathbf{x}_{r}$ of the solution of (6); see [21] for details. The application of the forward mode to $h$ yields the Taylor coefficients $y_{0}, \ldots, y_{r}$ of the associated output signal

$$
y(t)=y_{0}+y_{1} t+y_{2} t^{2}+\cdots+y_{r} t^{r}+O\left(t^{r+1}\right) .
$$

Comparing (7) and (12), we can express the Lie derivatives $L_{\mathbf{f}}^{i} h(\mathbf{x})$ in terms of Taylor coefficients calculated with automatic differentiation [22]:

$$
L_{\mathbf{f}}^{i} h(\mathbf{x})=i ! y_{i} \quad \text { for } \quad i=0, \ldots, r
$$

Similarly, one can compute the Taylor coefficients of the output of a second autonomous system

$$
\dot{\mathbf{x}}=\mathbf{f}(\mathbf{x})+\mathbf{g}(\mathbf{x}), \quad y=h(\mathbf{x})
$$

for $\mathbf{x}(0)=\mathbf{x}_{0}$ as well as the associated Lie derivatives $L_{\mathbf{f}+\mathbf{g}}^{i} h(\mathbf{x})$. Let $\bar{y}_{i}$ and $\tilde{y}_{i}$ denote the Taylor coefficients of the output of (6) and (14), respectively. Since the system (3) is assumed to have relative degree $r$, we have

$$
L_{\mathbf{f}}^{i} h(\mathbf{x})=i ! \bar{y}_{i}=i ! \tilde{y}_{i} \quad \text { for } \quad i=0, \ldots, r-1
$$

The mixed Lie derivative (5) can be obtained from

$$
L_{\mathbf{g}} L_{\mathbf{f}}^{r-1} h\left(\mathbf{x}_{0}\right)=L_{\mathbf{f}+\mathbf{g}}^{r} h\left(\mathbf{x}_{0}\right)-L_{\mathbf{f}}^{r} h\left(\mathbf{x}_{0}\right)=r !\left(\tilde{y}_{r}-\bar{y}_{r}\right) .
$$

Using (13), (15), and (16), we finally obtain an expression of the feedback (11) in terms of Taylor coefficients computed by automatic differentiation:

$$
u=\frac{1}{r !\left(\bar{y}_{r}-\tilde{y}_{r}\right)}\left[0 ! k_{0} \bar{y}_{0}+1 ! k_{1} \bar{y}_{1}+\cdots+(r-1) ! k_{r-1} \bar{y}_{r-1}+r ! \bar{y}_{r}-v\right]
$$


The methods derived here will be applied to two examples. Example 1 demonstrates the basic concepts. Actual performance data is presented in Example 2.

Example 1. Consider the state-space system

$$
\dot{\mathbf{x}}=\left(\begin{array}{c}
x_{1} x_{2}-x_{1}^{3} \\
x_{1} \\
-x_{3} \\
x_{1}^{2}+x_{2}
\end{array}\right)+\left(\begin{array}{c}
0 \\
2+2 x_{3} \\
1 \\
0
\end{array}\right) u, \quad y=x_{4}
$$

taken from [2, Ex. 4.1.5, 4.4.1]. Since

$$
\begin{aligned}
y & =x_{4} \\
\dot{y} & =\dot{x}_{4}=x_{1}^{2}+x_{2} \\
\ddot{y} & =2 x_{1} \dot{x}_{1}+\dot{x}_{2} \\
& =\underbrace{2 x_{1}\left(x_{1} x_{2}-x_{1}^{3}\right)+x_{1}}_{L_{\mathbf{f}}^{2} h(\mathbf{x})}+\underbrace{\left(2+2 x_{3}\right)}_{L_{\mathbf{g}} L_{\mathbf{f}} h(x)} u
\end{aligned}
$$

system (18) has relative degree $r=2$ everywhere except at $x_{3}=-1$. Table 1 shows the Lie derivatives and the Taylor coefficients computed at $\mathbf{x}=(1,2,3,4)^{T}$ with ADOL-C. The coefficients $\mathbf{x}_{i}$ have been calculated with forode. To get the Taylor coefficients of the output we used the $\mathrm{C}++$ function forward. From the entry in the last row and last column we obtain the mixed Lie derivative $L_{\mathbf{g}} L_{\mathbf{f}} h(\mathbf{x})=8$.

Table 1. Lie derivatives associated with Example 1

\begin{tabular}{ccccc}
\hline$i$ & $L_{\mathbf{f}}^{i} h(\mathbf{x})$ & $i ! \bar{y}_{i}$ & $i ! \tilde{y}_{i}$ & $i !\left(\tilde{y}_{i}-\bar{y}_{i}\right)$ \\
\hline 0 & $x_{4}$ & 4 & 4 & 0 \\
1 & $x_{1}^{2}+x_{2}$ & 3 & 3 & 0 \\
2 & $2 x_{1}^{2} x_{2}-2 x_{1}^{4}+x_{1}$ & 3 & 11 & 8 \\
\hline
\end{tabular}

To stabilize the example system (18), we used the feedback (17) with $v \equiv 0$. We have chosen the coefficients $k_{0}=1$ and $k_{1}=2$, i.e., the characteristic equation $\rho(\lambda)=k_{0}+k_{1} \lambda+\lambda^{2}=0$ has the roots $\lambda_{1}=\lambda_{2}=-1$. The trajectories of the closed-loop system, i.e., system (3) with the control (17), for the initial value $\mathbf{x}_{0}=\mathbf{x}(0)=(1,2,3,4)^{T}$ are shown in Fig. 2 . We used bold lines for the states $x_{1}, \ldots, x_{4}$, and a dashed line for the control $u$. Note that the trajectories converge to the origin. 


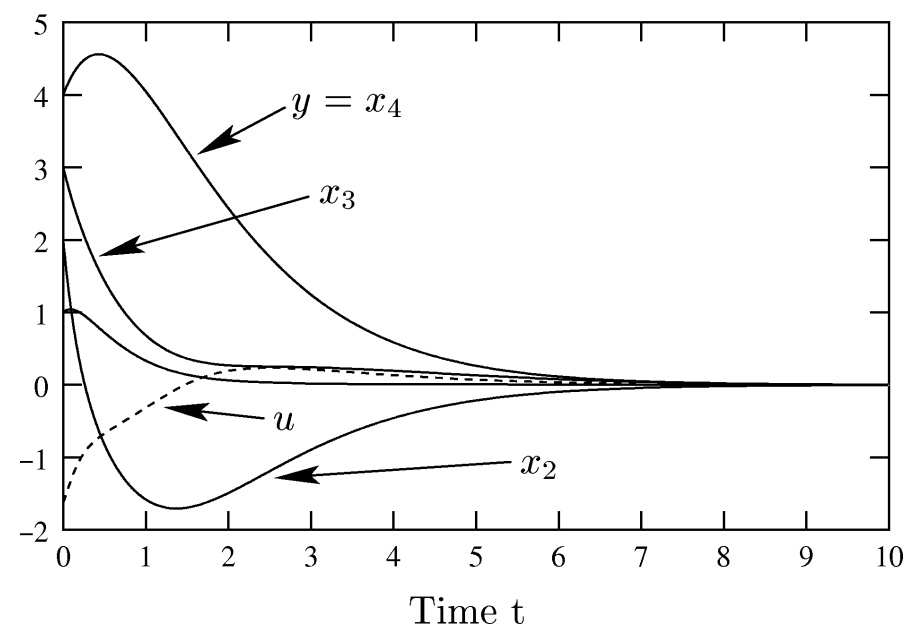

Fig. 2. Trajectories of the closed-loop example system for $0 \leq t \leq 10$

Example 2. The structure of the Translational Oscillator with Rotating Actuator (TORA) benchmark system [23, 24] is sketched in Fig. 3. The system consists of a platform of mass $M$ that can oscillate without damping in the horizontal plan. The platform is connected to a fixed frame by a linear spring with spring constant $k$. A rotating eccentric mass $m$ on the platform is actuated by a motor. Let $x_{1}$ and $x_{2}$ denote the (normalized) displacement and the velocity of the platform from the equilibrium position, respectively. Moreover, let $x_{3}$ and $x_{4}$ be the angle and the angular velocity of the rotor. In these coordinates, the state-space equations (3) are

$$
\begin{aligned}
& \dot{x}_{1}=x_{1} \\
& \dot{x}_{2}=\frac{-x_{1}+\varepsilon x_{4}^{2} \sin x_{3}}{1-\varepsilon^{2} \cos ^{2} x_{3}}+\frac{-\varepsilon \cos x_{3}}{1-\varepsilon^{2} \cos ^{2} x_{3}} u \\
& \dot{x}_{3}=x_{4} \\
& \dot{x}_{4}=\frac{\varepsilon \cos x_{3}\left(x_{1}-\varepsilon x_{4}^{2} \sin x_{3}\right)+u}{1-\varepsilon^{2} \cos ^{2} x_{3}} \\
& y=x_{1}
\end{aligned}
$$

where $u$ is the control torque applied to the rotor and $y$ is the output. The parameter $\varepsilon$ depends on the physical parameters mentioned above. We used the value $\varepsilon=0.1$.

We will compare the evaluation time of symbolically computed Lie derivatives $L_{\mathbf{f}}^{k} h\left(\mathbf{x}_{0}\right)$ for $k=1, \ldots, 10$ with the time required to obtain these derivatives using automatic differentiation. All tests were performed on a $700 \mathrm{MHz}$ Athlon PC under SuSE Linux 7.2. We used the GNU C++ compiler gcc 2.95.3.

The symbolic computation was carried out with the computer algebra package MuPAD 2.0 [25]. The Lie derivatives have been computed using (4). For 


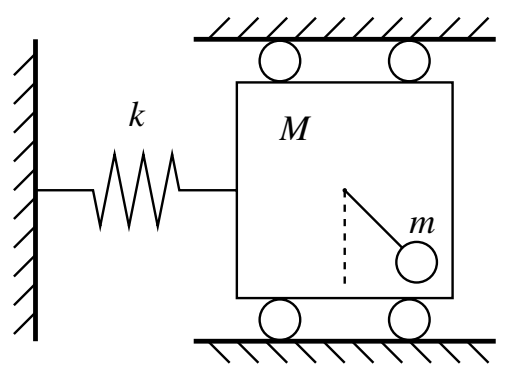

Fig. 3. TORA system configuration

example, the first Lie derivatives are

$$
L_{\mathbf{f}} h(\mathbf{x})=x_{2} \quad \text { and } \quad L_{\mathbf{f}}^{2} h(\mathbf{x})=\frac{-x_{1}+\varepsilon x_{4}^{2} \sin x_{3}}{1-\varepsilon^{2} \cos ^{2} x_{3}} .
$$

Subsequently, the results were converted to $\mathrm{C}$ code with the MUPAD command generate: : C. Depending on the order $k$ of the Lie derivatives, the size of the resulting $\mathrm{C}$ source code increases significantly, see Table 2. As shown in Fig. 4, the time to evaluate these expressions after compilation increases drastically with respect to the order of the derivatives involved.

Table 2. Size of the symbolically generated C source code

\begin{tabular}{lrrrrrrrrrr}
\hline Order $k$ of $L_{\mathbf{f}}^{k} h(\mathbf{x})$ & 1 & 2 & 3 & 4 & 5 & 6 & 7 & 8 & 9 & 10 \\
\hline Size in bytes & 13 & 73 & 295 & 852 & 2078 & 4420 & 8440 & 15018 & 25507 & 40600 \\
\hline
\end{tabular}

Moreover, we computed the function values $L_{\mathbf{f}}^{k} h\left(\mathbf{x}_{0}\right)$ by means of automatic differentiation as discussed in Sect. 4, i.e., the coefficients of the series expansion (7) have been computed with Taylor arithmetic. In our example, Fig. 4 shows that ADOL-C is faster than the symbolically generated code for $k \geq 6$, whereas TADIFF is faster for $k \geq 5$. For $k=10$, ADOL-C needs only 20 percent and TADIFF only 8.6 percent of the CPU time required to evaluate the symbolically generated Lie derivative $L_{\mathbf{f}}^{10} h\left(\mathbf{x}_{0}\right)$. In this application, TADIFF is approximately twice as fast as ADOL-C; but note that ADOL-C offers more functionality.

Nevertheless, the example system (19) is still very simple. For slightly more complicated example systems [26] the break even point between symbolic and automatic differentiation lies around $k=2$ or $k=3$. Full advantages of automatic differentiation can only be exploited for systems with more complicated right hand sides. 


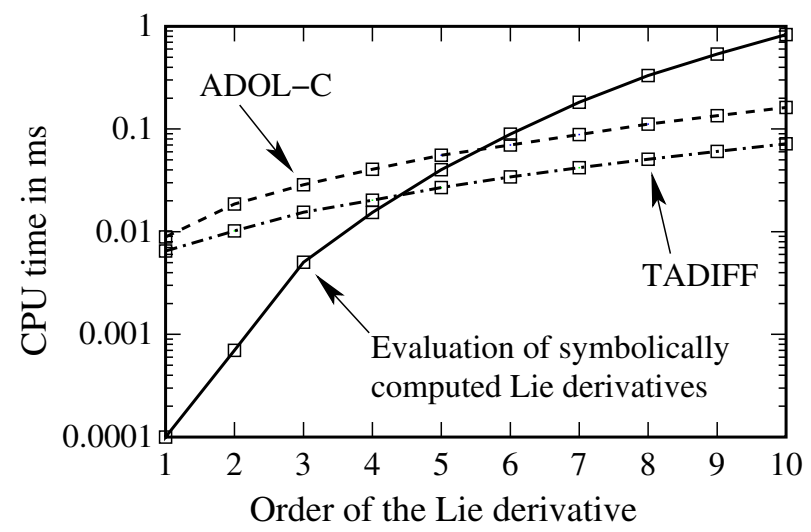

Fig. 4. CPU time needed for evaluation of Lie derivatives

\section{$5 \quad$ Asymptotic Output Tracking}

In this section we will discuss the use of automatic differentiation for tracking control. The aim is to design a controller such that the system's output $y$ converges asymptotically to a prescribed reference trajectory $y_{\text {ref }}$. We want to computed the feedback of an output tracking controller by means of automatic differentiation. From a control-theoretic point of view, the problem is addressed in [2, Sect. 4.5].

Imposing the input

$$
\begin{aligned}
u & =\frac{1}{L_{\mathbf{g}} L_{\mathbf{f}}^{r-1} h(\mathbf{x})}\left[-\sum_{i=0}^{r-1} k_{i}\left(L_{\mathbf{f}}^{i} h(\mathbf{x})-y_{\text {ref }}^{(i)}-L_{\mathbf{f}} h(\mathbf{x})^{r}+y_{\mathrm{ref}}^{r}\right)\right] \\
& =\frac{1}{L_{\mathbf{g}} L_{\mathbf{f}}^{r-1} h(\mathbf{x})}\left[k_{0}\left(y_{\mathrm{ref}}-y\right)+\cdots+k_{r-1}\left(y_{\text {ref }}^{(r-1)}-y^{(r-1)}\right)+\left(y_{\text {ref }}^{(r)}-y^{(r)}\right)\right],
\end{aligned}
$$

the error function $e(t)=y(t)-y_{\text {ref }}(t)$ satisfies the linear differential equation

$$
k_{0} e+k_{1} e^{(1)}+\cdots+k_{r-1} e^{(r-1)}+e^{(r)}=0
$$

If the coefficients $k_{0}, \ldots, k_{r-1}$ are chosen in such a way that all roots of the associated characteristic polynomial are in the open complex left half plane, the error converges to zero, i.e., $e(t) \rightarrow 0$ for $t \rightarrow \infty$. This means that the real output converges to the reference output, i.e., $y(t) \rightarrow y_{\text {ref }}(t)$ for $t \rightarrow \infty$.

Again, the controller feedback (20) can very efficiently be computed using automatic differentiation. Let $\bar{y}_{i}$ and $\tilde{y}_{i}$ denote the $i$ th Taylor coefficient of (6) and (14), respectively, computed as described in Sect. 4. The Taylor coefficients $\breve{y}_{0}, \ldots, \breve{y}_{r}$ of $y_{\text {ref }}(\cdot)$ can be obtained directly using Taylor arithmetic (see Sect. 2). 
In terms of these Taylor coefficients, the feedback (20) can be written as

$$
\begin{aligned}
u= & \frac{1}{r !\left(\bar{y}_{r}-\tilde{y}_{r}\right)}\left[k_{0}\left(\bar{y}_{0}-\breve{y}_{0}\right)+k_{1}\left(\bar{y}_{1}-\breve{y}_{1}\right)+2 k_{2}\left(\bar{y}_{2}-\breve{y}_{2}\right)+\cdots\right. \\
& \left.+(r-1) ! k_{r-1}\left(\bar{y}_{r-1}-\breve{y}_{r-1}\right)+r !\left(\bar{y}_{r}-\breve{y}_{r}\right)\right] \\
= & \frac{1}{r !\left(\bar{y}_{r}-\tilde{y}_{r}\right)} \sum_{i=0}^{r} i ! k_{i}\left(\bar{y}_{i}-\breve{y}_{i}\right)
\end{aligned}
$$

with $k_{r}=1$.

\section{Conclusions}

The controller design by exact linearization via nonlinear feedback has been considered. The author presented a method to compute the state feedback of the controller using automatic differentiation. The method is applicable to systems with smooth but complicated nonlinearities. One advantage is the fact, that the system to be controlled must not be given by an explicit symbolic formula but by an algorithm. An other advantage is that higher order derivatives can be computed very fast with automatic differentiation. Moreover, the method presented here is well-suited for rapid prototyping in system design.

\section{References}

1. Jakubczyk, B., Respondek, W., Tchoń, K., eds.: Geometric Theory of Nonlinear Control Systems. Wroclaw Technical University Press (1985)

2. Isidori, A.: Nonlinear Control Systems: An Introduction. 3rd edn. Springer (1995)

3. Nijmeijer, H., van der Schaft, A.J.: Nonlinear Dynamical Control Systems. Springer (1990)

4. Joo, S., Seo, J.H.: Design and analysis of the nonlinear feedback linearizing control for an electromagnetic suspension system. IEEE Trans. on Control Systems Technology 5 (1997) 135-144

5. Bolzern, P., DeSantis, R.M., Locatelli, A., Masciocchi, D.: Path-tracking for articulated vehicles with off-axle hitching. IEEE Trans. on Control Systems Technology 6 (1998) 515-523

6. Fujimoto, K., Sugie, T.: Freedom in coordinate transformation for exact linearization and its application to transient behaviour improvement. Automatica 37 (2001) 137-144

7. Rothfuss, R., Zeitz, M.: Einführung in die Analyse nichtlinearer Systeme. In Engell, S., ed.: Entwurf nichtlinearer Regelungen. Oldenbourg-Verlag (1995) 3-22

8. Kugi, A., Schlacher, K., Novaki, R. In: Symbolic Computation for the Analysis and Synthesis of Nonlinear Control Systems. Volume IV of Software for Electrical Engineering, Analysis and Design. WIT-Press (1999) 255-264

9. Kwatny, H.G., Blankenship, G.L.: Nonlinear Control and Analytical Mechanics: A Computational Approach. Birkhäuser (2000)

10. de Jager, B.: The use of symbolic computation in nonlinear control: is it viable? IEEE Trans. on Automatic Control AC-40 (1995) 84-89 
11. Grimm, C., Meise, C., Oehler, P., Waldschmidt, K., Fey, F.: AnalogSL: A library for modeling analog power drivers with C++. Forum on Design Languages FDL'01, September 3-7, 2001, Lyon, France (2001)

12. Tummescheit, H.: Object-oriented modeling of physical systems with Modelica. Short Tutorial (2000)

13. Clauß, C., Schneider, A., Schwarz, P.: Objektorientierte Modellierung physikalischer Systeme, Teile 13 und 14. Automatisierungstechnik (2000)

14. Haase, J., Schwarz, P., Trappe, P., Vermeiren, W.: Erfahrungen mit VHDL-AMS bei der Simulation heterogener Systeme. In: 3. ITG/GI/GMM-Workshop "Methoden und Beschreibungssprachen zur Modellierung und Verifikation von Schaltungen und Systemen", February 28-29, 2000, Frankfurt, Germany. (2000) 167-175

15. Klein, W., Griewank, A., Walther, A.: Differentiation methods for industrial strength problems. In Corliss, G., Faure, C., Griewank, A., Hascoët, L., Naumann, U., eds.: Automatic Differentiation of Algorithms: From Simulation to Optimization. Springer (2002) (to appear).

16. Griewank, A., Juedes, D., Utke, J.: ADOL-C, a package for the automatic differentiation of algorithms written in $\mathrm{C} / \mathrm{C}++$. ACM Transactions on Mathematical Software 22 (1996) 131-167

17. Bendtsen, C., Stauning, O.: TADIFF, a flexible $\mathrm{C}++$ package for automatic differentiation. Technical Report IMM-REP-1997-07, TU of Denmark, Dept. of Mathematical Modelling, Lungby (1997)

18. Griewank, A.: Evaluating Derivatives: Principles and Techniques of Algorithmic Differentiation. SIAM, Philadelphia (2000)

19. Marino, R., Respondek, W., van der Schaft, A.J.: Equivalence of nonlinear systems to input-output prime forms. SIAM J. Control and Optimization 32 (1994) 387407

20. Kailath, T.: Linear Systems. Prentice-Hall (1980)

21. Griewank, A.: ODE solving via automatic differentiation and rational prediction. In Griffiths, D.F., Watson, G.A., eds.: Numerical Analysis 1995. Volume 344 of Pitman Research Notes in Mathematics Series. Addison-Wesley (1995)

22. Röbenack, K., Reinschke, K.J.: Reglerentwurf mit Hilfe des Automatischen Differenzierens. Automatisierungstechnik 48 (2000) 60-66

23. Wan, C.J., Bernstein, D.S., Coppola, V.T.: Global stabilization of the oscillating eccentric rotor. In: Proc. 33rd IEEE Conf. Decision and Contr. (1994) 4024-4029

24. Bupp, R.T., Wan, C.J., Cappola, V.T., Bernstein, D.S.: Design of a rotational actuator for global stabilization of translational motion. In: Proc. Symp. Active Contr. Vibration Noise, ASME Winter Mtg. (1994)

25. Oevel, W., Postel, F., Rüscher, G., Wehrmeier, S.: Das MuPAD Tutorium. Springer (1999) Deutsche Ausgabe.

26. Röbenack, K., Vogel, O.: Numerische Systeminversion. Automatisierungstechnik 48 (2000) 487-495 